\title{
Formulaic expressions in language technology
}

\author{
María Fernández-Parra
}

Swansea University, United Kingdom

https://doi.org/10.36505/ExLing-2008/02/0024/000083

\begin{abstract}
Little attention has been given to the treatment of formulaic expressions in language technology during the past few decades, although such expressions are in fact extremely common both in conversation and in written discourse. Computer-Assisted Translation (CAT) tools are currently the most widely used language technology tools among professional translators. In this paper, I intend to determine the usefulness of such tools in the treatment of formulaic expressions. In particular, the aim is to evaluate the performance of Trados (2007 version), the leader in the CAT-tools market, in the treatment of such expressions, compared to the treatment of terms and ordinary translation units, by examining the performance of selected components in the identification and translation of formulaic expressions.
\end{abstract}

Key words: computer-assisted translation, Trados, formulaic language

\section{Formulaic expressions and Trados}

Formulaic expressions are understood here as consisting of prefabricated expressions, that is to say "stored and retrieved whole" (Wray 2002: 9) from the mental lexicon at the time of use. Idioms (e.g. rock the boat, spill the beans), proverbs (e.g. a stitch in time saves nine) and collocations (e.g. auspicious event, teething problem) can be included as subclasses of formulaic language, but formulaic language also includes expressions that would not usually be considered idioms, such as good morning, money talks, first thing tomorrow, etc.

As formulaic expressions are common and pervasive (cf. Jackendoff 1997:156), they constitute challenges for the professional translator, who is bound by constant tight deadlines. Translators may be well versed in the translation of terms, but not to the same degree in the translation of formulaic expressions. An analogy can be established in that both formulaic expressions and terms can be single or multiword units. Since Trados has been proven to perform well in the treatment of terms, it could theoretically perform well also in the treatment of formulaic expressions.

\section{Identification of formulaic expressions}

In Trados, Workbench works as a central platform during translation, drawing on the Translation Memory (TM) and the termbase (TB). Another component of Trados, Multiterm Extract, has functions that feed into the termbase as well, such as Monolingual Extraction. Workbench searches the

ExLing 2008: Proceedings of 2nd Tutorial and Research Workshop on Experimental Linguistics, 25-27 August 2008, Athens, Greece 
source text for already existing terms in the Multiterm termbase, so that the translator can consult all their previous translations and any associated information about them, whereas the Monolingual Extraction tool searches the source text for new terms, so that they may be added to the termbase. Similarly, both identification methods may be used for formulaic expressions.

A selected source text of about 10,000 words was used to determine the usefulness of Workbench and Monolingual Extraction to identify formulaic expressions. First of all, the text was searched manually and 72 formulaic expressions were identified, with 89 tokens. Then, the search was repeated with both Workbench and Monolingual Extraction and the results compared.

Only one token per formulaic expression was counted in the Monolingual Extraction project. No additional tokens were counted, as formulaic expressions need only be identified once, in order to be included in the termbase. In Workbench, however, the identification of each token of a formulaic expression is essential, as the link to its entry in the termbase is only established automatically if identification of the string has taken place. If identification fails, the translator can look up the termbase manually, but at the expense of valuable time.

\section{Workbench translation memory}

The main problems encountered by Workbench when trying to identify specific segments in a text are variation and noise. In this exercise, two main types of variation were found, inflectional variation and word order inversion. Although fuzzy settings may be altered in Workbench, in this exercise the lowest fuzzy settings possible were chosen, in order to obtain the highest number of identified expressions, on one hand, and, on the other hand, in order to try to overcome the problem of variation, which may be more acute for formulaic expressions than for terms.

Overall, the results obtained with Workbench are promising. Workbench identified 65 tokens of formulaic expressions, out of a maximum of 89 . Workbench also identified a further 15 tokens of formulaic expressions that had not been identified manually. In total, therefore, 80 tokens were identified with Workbench. By contrast, Workbench never managed to recognise 13 of the expressions that were found manually.

The most common types of inflectional variation in this exercise were tense variations and singular/plural variations. It would appear that, for Workbench, tense variations are bigger obstacles in the recognition of terms/formulaic expressions than singular/plural variations. It recognised all tokens of expressions with singular/plural variations but only half of the tokens displaying tense variations. For example, it recognised the expression raise money in the string First, more money must be raised, but it did not 
recognise the expression keep pace with in the string ... and middle income countries in all regions has not kept pace with the need for expanded.... A possible solution might be to include, as an index field in the termbase, those forms of the expression which differ most from the canonical form, in order to possibly facilitate their recognition by Workbench.

As for word order inversion, Workbench identified 6 out of the 16 occurrences of expressions with inverted word orders. For example, defying noise, word order and variation, Workbench identified the expression make a contribution in the string Funding is only one component of the significant contribution the public sector makes to HIV vaccine....

Noise was another factor to take into account. From the total of 89 occurrences, 30 appeared with varying degrees of noise, out of which Workbench identified 16. For example, Workbench identified the expression meet a standard from the string In countries that meet public expenditure management standards, aid flows through government... Without taking any of these figures as absolute, they nevertheless indicate that the trend in identifying formulaic expressions with Workbench is a successful one overall.

\section{Monolingual extraction tool}

The Monolingual Extraction tool differs from Workbench in that it produces an editable list of 'candidate' terms, each of which may or may not be selected by the translator for inclusion in the termbase. Because the candidate term strings may contain any portion of source text, not only terms, the Monolingual Extraction tool could theoretically be used to identify formulaic expressions 'embedded' in the candidate term.

The extraction settings were set at a minimum of 2 words per expression, since one-word formulaic expressions are not of the same computational interest as expressions of 2 words or more. Further, a maximum of 4 words per expression was also set, having established during the manual search that no expression was longer than 4 words. In a Monolingual Extraction project, it is also possible to choose from a wide range of noise level settings, from no noise at all (level 0 ) to maximum noise (level 1), including every noise level from 0.1 to 0.9 in between. The search for formulaic expressions was performed at each of these levels.

The results obtained with Monolingual Extraction were also promising. From a total of 72 formulaic expressions found manually, the program identified 59, and an additional 18 which had not been identified manually. Like Workbench, Monolingual Extraction did not recognise the same 13 expressions that were only identified manually. With a total of 77 expressions identified, therefore, the Monolingual Extraction tool identified more formulaic expressions in total than were identified manually. 
The main obstacle in the identification of formulaic expressions appears to be noise rather than variation, as formulaic expressions were identified regardless of inflectional variation and word order inversion. More noise means that more formulaic expressions can be identified, but more time will be needed to search through long lists of candidate terms. Without noise, very few formulaic expressions, and indeed terms, were identified by the machine. In fact, in order to identify a decent number of formulaic expressions, the best noise settings turned out to be 0.8 .

\section{Conclusions}

From the discussion in the previous sections, it can be initially concluded that both Workbench and Monolingual Extraction perform as well in the identification of formulaic expressions as they already do in the identification of terms. Both Workbench and Monolingual Extraction recognised the majority of formulaic expressions in the source text and both tools identified tokens of formulaic expressions that had not been identified manually.

The best results in the identification of formulaic expressions with Workbench are obtained with the lowest possible fuzzy settings, which may not always be the case with terms. Also, variation appears to be a bigger obstacle than noise for Workbench to recognise certain formulaic expressions. A possible solution to consider might be the inclusion of additional index fields to certain entries in the termbase containing inflected forms of the expression that differ considerably from the generic form.

The best results in the identification of formulaic expressions with Monolingual Extraction were obtained with the higher levels of noise. In these settings, a large amount of formulaic expressions were identified. However, in order to get good results, the translator has to search through long lists of candidate terms. A possible solution to contend with the large amount of noise would be a compromise, namely, to spend the time searching through the long lists of candidate terms only when the number of occurrences of formulaic expressions in a given source text seems to warrant it.

To sum up, Trados offers very different functions that can be used in the identification of formulaic expressions, as well as terms. The combined results obtained both with Workbench and Monolingual Extraction can support the translator significantly.

\section{References}

Jackendoff, R. 1997. The Architecture of the language faculty. Cambridge, MA: Newbury.

Wray, A. 2002. Formulaic language and the lexicon. Cambridge: CUP. 